\title{
Spectroscopy of New Planetary Nebulae Close to the Galactic Center
}

\author{
G.C. Van de Steene ${ }^{1}$ and G.H. Jacoby ${ }^{2}$ \\ ${ }^{1}$ European Southern Observatory; ${ }^{2}$ Kitt Peak National Observatory
}

Planetary Nebulae (PN) are bright emission line objects, observable at large distances throughout the Galaxy. They serve as probes of abundance gradients and chemical enrichment history of the ISM.

There has been a lack of PN within 2 degrees of the galactic plane, especially in the direction of the bulge. This lack is not due to disinterest in such objects, but rather the severe extinction towards the bulge accounts for this.

The following reasons motivated our search for many new PN in the Galactic Bulge region: 1) Within 2 degrees of the galactic center, only $34 \mathrm{PN}$ are known, but $\sim 200$ are expected based on the PN luminosity function and the distribution of OH/IR stars, precursors of PN (Pottasch, priv. communication). 2) $90 \%$ of small PN within 10 degrees of the galactic center are actually close to it (Pottasch \& Acker 1989, A\&A 221, 123). If we find PN they provide keys to the stellar population of the Bulge. 3) It is implied that bulge PN derive only from the low end of the metallicity range in metal-rich populations (Ferguson \& Davidsen, 1993, ApJ 408, 92) since stars at the high end have greater mass loss and never ascend the AGB. We can test this hypothesis directly. 4) If the bulge consists of a population having a large range in age and metallicity, we would expect a spatially dependent abundance gradient. PN abundances throughout the bulge provide a direct test for an age dispersion in PN progenitors. 5) Bulge kinematics are trivial to measure very accurately with PN, and may help map out the extent of a central bar. 6) These PN can be assumed to be all at the same distance $(\sim 8 \mathrm{kpc})$, and so we can derive their distancedependent parameters (mass, luminosity, temperatures, age) that are crucial for studying the underlying population.

We imaged a 4 x 4 degree field around the galactic center in [S III] $\lambda 9532$. This survey revealed 95 new PN candidates in addition to the 34 previously known in this region (Acker et al. 1992, Catalogue of galactic planetary nebulae, ESO; Kohoutek 1994, Astron. Nachr. 315, 235). Optical spectra were obtained with the $1.52 \mathrm{~m}$ ESO telescope in 1995 and 1996. All but one of the 46 new PN candidates observed spectroscopically showed optical emission line spectra typical of PN. The velocity dispersion of $92 \mathrm{~km} / \mathrm{s}$ is consistent with the stellar velocity dispersion in the bulge and reaffirms the likelihood that these PN are bulge, rather than foreground objects. There doesn't seem to be contamination by $\mathrm{H}$ II regions based on the IRAS data. NTT images obtained for $10 \mathrm{PN}$ indicate typical PN sizes between 0.05 and $0.28 \mathrm{pc}$ at a distance of $7.8 \mathrm{kpc}$.

We have made a first attempt to calculate abundances for 5 new PN having the richest spectra. A modified version of the photo-ionization code CLOUDY is used to calculate various models, searching for a best fit of the predictions of physical parameters to the observables (optical emission lines and angular diameter) (van Hoof \& Van de Steene, 1997, A\&A submitted). Abundances indicate that three PN are somewhat metal-poor having $[\mathrm{Ar} / \mathrm{H}]=-0.3$ relative to disk PN (Clegg, IAU Symp. $155 \mathrm{PN}, \mathrm{p} .553$ ), while the other two have $[\mathrm{Ar} / \mathrm{H}]$ similar to disk $\mathrm{PN}$. 